\title{
On the theory of magnetoviscous effect in magnetorheological suspensions
}

\author{
A. Zubarev and L. Iskakova
}

Urals Federal University, Lenina Ave 51, 620083, Ekaterinburg, Russia

\author{
M.T. López-López \\ Departamento de Física Aplicada, Facultad de Ciencias, Universidad de Granada, Campus de \\ Fuentenueva, 18071 Granada, Spain. \\ P. Kuzhir and G. Bossis
}

CNRS, UMR7336, Laboratoire de Physique de la Matière Condensée, Université de Nice-Sophia Antipolis, 28 avenue Joseph Vallot, 06100 Nice, France.

\section{Synopsis}

A theoretical model of magnetoviscous effect in a suspension of non-Brownian linearly magnetizable particles is suggested. A simple shear flow in the presence of an external magnetic field aligned with the velocity gradient is considered. Under the action of the applied field, the particles are supposed to form dense highly elongated drop-like aggregates. Two different scenarios of the aggregates' destruction under shearing forces are considered, namely a "bulk" destruction of aggregates into pieces, and an "erosive" destruction connected to the rupture of individual particles from the aggregate surface. Both models are based on a balance of forces acting either on the whole aggregate or on individual particles. The two approaches lead to qualitatively different Mason number $(M a)$ behaviors of the magnetic suspensions: the suspension viscosity scales as either $M a^{-2 / 3}$ for the bulk destruction of aggregates or $M a^{-4 / 5}$ for the erosive destruction. In any case, we do not recover Bingham behavior $\left(M a^{-1}\right)$ often predicted by chain models of the magneto- or electrorheology. Our theoretical results are discussed in view of comparison with existing theories and experimental results in the wide range of Mason numbers. 


\section{Introduction}

Suspension of magnetizable particles in a liquid, so-called magnetorheological suspensions (MRS), attract considerable interest of investigators and engineers due to a rich set of physical properties which find broad applications in many modern technologies. One of the most interesting features of these systems is their ability to change, up to several orders of magnitude, their rheological properties under the action of quite moderate magnetic fields [Shulman and Kordonski (1982), Ginder (1998), Bossis et al. (2002), Odenbach (2002), Bayat et al. (2009), de Vicente et al. (2011)].

The physical cause of the strong rheological effects is assembling of the particles in heterogeneous structures strongly aligned along the applied field. A number of different structures has been observed and/or predicted in magnetic suspensions. In the quiescent state, a hexagonal pattern of column-like aggregates is most often observed in the MRS sandwiched between two parallel plates and subjected to a stationary magnetic field perpendicular to the plates [Liu et al. (1995)]. An arrangement of particles inside the columns into a body-centeredtetragonal (BCT) lattice has been proved to be the most favorable from the energy viewpoint [Tao and Sun (1991)]. However, smallness of thermal fluctuations, non-negligible gravitational forces and eventual solid friction between particles may hinder thermodynamic equilibrium states and lead to less organized structures, such as branched chains [Klingenberg et al. (1991)], zigzag chains [Bossis et al. (1997)] or irregularly shaped aggregates [Bonnecaze and Brady (1990)]. In flowing suspensions these structures may become more complicated but the suspension still preserves its high anisotropy with respect to the field and the flow orientation so that, at low-to-moderate concentrations, the structure can still be modeled as an ensemble of parallel chains [Shulman et al. (1982), Martin and Anderson (1996)] or dense drop-like [Halsey et al. (1992), Shulman et al. (1984)] or cylindrical [Gomez-Ramirez et al. (2011)] aggregates. Note that regular sheet-like patterns have been observed in suspensions of smaller magnetic polystyrene particles undergoing a week Brownian motion, both at steady [Volkova et al. (1999)] 
and oscillatory shear [Cutillas et al. (1998)]. However, to our knowledge, such shear-banding has not been reported for conventional MRS composed of polydisperse large iron particles. More recently, honeycomb and foam-like structures were generated with the help of triaxial magnetic fields in view of application to composite materials [Martin et al. (2004)].

Under shear flow, the field-induced particle structures enhance the suspension stress level and often lead to a Bingham like rheological behavior:

$$
\sigma=\eta_{0} \dot{\gamma}+\sigma_{a}
$$

Here and below $\sigma$ is the macroscopic (measured) stress in the suspension, $\eta_{0}$ is the viscosity of the carrier liquid, $\dot{\gamma}$ is the macroscopic shear rate, $\sigma_{a}$ is the stress produced by the aggregates. The relation (1) has been derived mathematically strictly (see, for example [Batchelor (a) (1970), Pokrovskii (1978)]) for dilute suspensions where the hydrodynamic interaction between aggregates and between separated particles is negligible. For systems where this interaction is significant, the high-shear viscosity, equal to the viscosity of the suspension of non-aggregated particles, can be used instead of $\eta_{0}$ [Berli and de Vicente (2012), Marshal et. al. (1989)]. Below, for maximal simplification of the mathematical part of the work, we will neglect any interaction between aggregates and will use relation (1).

From the scaling arguments, the only dimensional groups that can constitute the aggregate stress of non-Brownian suspensions are either the fluid stress $\eta_{0} \dot{\gamma}$ or the magnetostatic energy density, $\mu_{0} H^{2}$, or their combination, the simplest of which is the power law: $\frac{\sigma_{a}}{\eta_{0} \dot{\gamma}} \propto\left(\frac{\mu_{0} H^{2}}{\eta_{0} \dot{\gamma}}\right)$, with $\mu_{0}$ being the magnetic permeability of vacuum, $H$ - the macroscopic magnetic field inside the suspension and $v$ - a real number. Existing experimental data confirm this scaling but give rather dispersed values of the exponent $v$, varying in the range $0.7<v<0.95$, depending on the considered system (electrorheological fluids [Halsey et al. (1992)], MRS [Volkova et al. (2000), Klingenberg et al. (2007)], inverse ferrofluids [de Gans et al. (1999), Ramos et al. (2011)]). 
From the theoretical point of view, the aggregate stress $\sigma_{a}$ is determined by the concentration, size and shape of the aggregates. In their turn, in non-Brownian suspensions, these magnitudes are determined by the combination of the force of magnetic attraction between the particles, by the hydrodynamic forces which destroy the aggregates, and, eventually by the capillary forces tending to minimize the aggregate surface, thus contracting the aggregates.

In what follows, we shall briefly review the MRS rheological behavior predicted by the two most popular concepts of chain-like and drop-like aggregates, and then cite some other theoretical approaches. Theoretically, the effect of the chains on the stationary viscous properties of MRS with non-Brownian magnetizable particles has been studied by Shulman and Kordonski (1982) and, in details, by Martin and Anderson (1996). In the model of Martin and Anderson (1996), the stress $\sigma_{a}$ does not depend on the shear rate $\dot{\gamma}$ and, under the assumption of the linear magnetization of the particles, $\sigma_{a}$ is proportional to $\mu_{0} H^{2}$. The independence of the aggregate stress on the shear rate can be briefly explained in the following manner. The increasing shear flow progressively destroys the particle chains such that their length (or aspect ratio $r$ ) decreases with the shear rate as $r \propto \dot{\gamma}^{-1 / 2}$. The hydrodynamic stress generated on chains is proportional to the fluid stress $\eta_{0} \dot{\gamma}$ times the aspect ratio squared: $\sigma_{a} \propto \eta_{0} \dot{\gamma} r^{2}$. Therefore, the shear rate works out from the aggregate stress in the model of Martin and Anderson (1996) and gives rise to the dynamic yield stress. Some modifications to this approach can be found elsewhere [see, for example, de Gans et al. (1999), Volkova et al. (2000)]. Generalization of the chain model to the systems with the Brownian magnetizable particles has been done by Martin (2000) and Zubarev et al. (2007). It was shown that the Brownian effects lead to an increase of the stress $\sigma_{a}$ with $\dot{\gamma}$. However, for typical magnetic suspensions, composed of micron-sized particles, the energy of magnetic interaction between particles appears to be significantly larger than the thermal energy $k T$ at magnetic fields as low as $1 \mathrm{kA} / \mathrm{m}$. Therefore, Brownian effects can be ignored. The effect of the bulk "drops", consisting of a very large number of non-Brownian particles, has been 
studied by Halsey et al. (1992), Shulman et al. (1984), Kordonsky et al. (1990). The model of Halsey et al. (1992) is based on finding of extremum of the magnetic (electrical) free energy of a magnetizable (polarizable) ellipsoidal drop tilted with respect to the applied field. For the highly elongated drops the results lead to the following scaling relation $\sigma_{a} \propto\left(\mu_{0} H^{2}\right)^{2 / 3}\left(\eta_{0} \dot{\gamma}\right)^{1 / 3}$. It should be noted that the approach of the model of Halsey et al. (1992) does not take into account any mechanisms of the drop destruction by the action of the hydrodynamic viscous forces. Analysis shows that account of these mechanisms is principally important for development of a physically correct theory of rheological properties of suspensions with the heterogeneous aggregates.

The rupture of the particles from the surface of the "drop" by the viscous forces has been considered by Shulman et al (1984), Kordonsky et al. (1990). This model leads to the relation $\sigma_{a} \propto \mu_{0} H^{2}$; the stress $\sigma_{a}$ does not depend on the shear rate $\dot{\gamma}$ similarly to the chain model of Martin and Anderson (1996). The model of Shulman et al. (1984) and of Kordonsky et al. (1990) is often used for the interpretation of rheological effects in magnetic suspensions (see overview of Bossis et al. (2002)). However, this model contains a parameter $\Delta$ (the thickness of the gap between neighboring particles in the aggregates) which cannot be determined theoretically; it is considered as an empirically adjustable parameter. Strictly speaking, the gap thickness $\Delta$ can depend both on the field $H$ and the shear rate $\dot{\gamma}$, however in the model of Shulman et al (1984) and Kordonsky et al. (1990), the thickness $\Delta$ is considered as a constant. Analysis shows that this simplification plays a principle important role for the final results of the model. If we take into account a dependence of $\Delta$ on $\dot{\gamma}$ and $H$, the stress $\sigma_{a}$ will depend on $\dot{\gamma}$, i.e. the main result that $\sigma_{a}=$ const may be changed qualitatively. More recently, Gomez-Ramirez et al. (2011) have extended the Shulman's et al. model to magnetic fiber suspensions assuming aggregates of cylindrical shape and considering the aggregate break-up in its middle plane instead of rupture of individual particles from the aggregate surface. This model gave the same Bingham behavior 
$\sigma_{a} \propto \mu_{0} H^{2}$ but, when applied to a suspension of spherical particles, it under-predicted significantly the yield stress value.

An alternative particle-level approach was developed by Baxter-Drayton and Brady (1996) who related the escape time (required for particles trapped into a cage formed by their close neighbors to jump from cage to cage) to the suspension stress and shear rate. This theory gives a more sophisticated scaling for the aggregate stress but the results can be successfully fitted to the power-law scaling with the exponent $v$ varying between 0.9 and 1 at the dipolar coupling parameter (the ratio of magnetic-to-thermal energy) $2.5<\lambda<17$. Unfortunately, the theory invokes an adjustable parameter and the results for higher values of $\lambda$ (typically $10^{3}-10^{6}$ for conventional MRS) have not been reported. Nevertheless, Stokesian dynamics simulations of the same authors confirm this scaling for $10^{2}<\lambda<10^{12}$. More recently, a structural viscosity model has been proposed by Berli and de Vicente (2012) based on the balance between build up (magnetic field-induced clustering) and breakdown (shear-induced breakup) of particle aggregates. This theory predicts a shear-thinning behavior with a low and a high shear Newtonian plateaus and an intermediate region fitted to the power-law trend with the exponent $v$ lower than unity and depending on the ratio of high-shear-to-low-shear viscosity. This model may perfectly describe different experimental data at the expense of two adjustable parameters.

Summarizing, one can note that, in spite of the long time since the beginning of the theoretical study of the rheological properties of magneto- and electrorheological fluids, a satisfactory theory based on correct physical considerations, free of adjustable parameters and, at the same time, giving a satisfactory agreement with experiments, has not yet been developed. In the present paper, we present a model of the rheological properties of a suspension of magnetizable particles united in bulk drop-like aggregates. The model is based on the analysis of hydrodynamic and magnetic forces acting on the aggregates and does not use any adjustable parameter. We also compare our theoretical results with the experimental ones found in literature and with our own experimental results. 


\section{Theoretical model and main approximations}

\section{A. Torque equilibrium}

We consider a suspension, consisting of linearly magnetizable spherical particles. The typical size of the particles in modern MRS is about one micron. For particles of this size the Brownian effects are very weak, that is why we will neglect them. We will suppose that the particles are gathered in dense highly elongated "drops", considered as prolate ellipsoids of revolution with a major semi-axis $a$ and a minor semi-axis $b$ (Fig.1). Each drop consists of a very large number of particles. For maximal simplification of calculations, we restrict ourselves by the consideration of suspensions with highly elongated drops, in a similar way as in the works of Halsey et al. (1992), Shulman et al. (1984), Kordonsky et al. (1990).

The macroscopic stress $\sigma_{a}$ is determined by the volume concentration $\Phi$ of the drops in the suspension, by the angle $\theta$ of the drop deviation from the applied field $H$ and by the aspect ratio $r=a / b$ (see, for example, Halsey et al. (1992), Shulman et al. (1984), Kordonsky et al. (1990)).
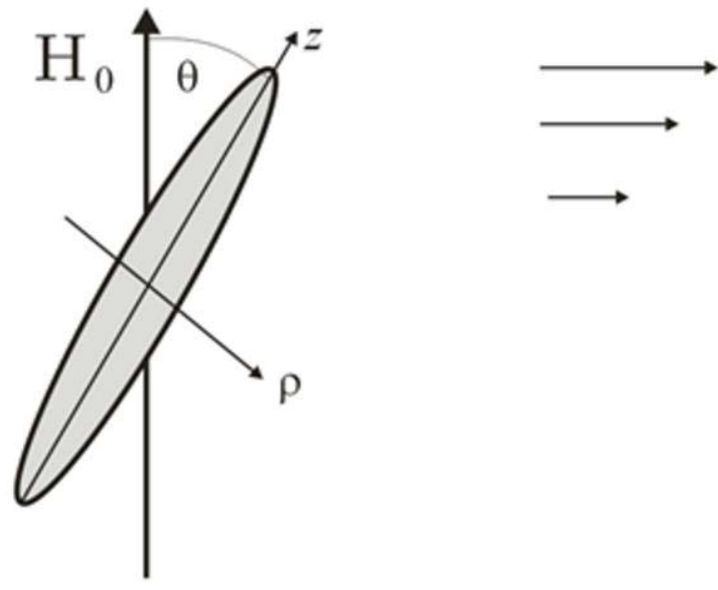

Fig.1. Sketch of the drop-like aggregate subjected to the shear and magnetic fields.

The volume concentration of drop-like aggregates in the suspension can be estimated from the obvious relation: 
$\Phi=\frac{\varphi_{0}}{\varphi_{a}}$

where $\varphi_{0}$ is the total volume concentration of the particles in the suspension, $\varphi_{a}$ is the concentration of the particles inside the drop. Since the energy of magnetic attraction between the particles in modern MRS is as a rule several orders of magnitude more than the thermal energy $k T$ [Bossis et al. (2002)], the concentration $\varphi_{a}$ inside the drop is very close to the concentration of the maximal packing. Therefore we can use the estimate $\varphi_{a} \approx 0.64$ for the random close packing limit.

The angle $\theta$ is determined by the balance between the magnetic and hydrodynamic torques, acting on the drop, which in the limit of highly elongated $(r>>1)$ and non-rotating aggregates reads [Halsey et al. (1992), Shulman et al. (1984), Kordonsky et al. (1990)]:

$$
\frac{1}{2} V \frac{\chi^{2}}{2+\chi} \mu_{0} H^{2} \sin 2 \theta=2 V \eta_{0} \dot{\gamma} \frac{r^{2}}{\ln r} \cos ^{2} \theta
$$

giving the following expression for the aggregate inclination angle $\theta$.

$$
\tan \theta=M a \frac{2(2+\chi)}{\chi^{2}} \frac{r^{2}}{\ln r}
$$

Here $\chi$ and $V$ are the magnetic susceptibility and the volume of the drop, respectively, and $M a=\eta_{0} \dot{\gamma} /\left(\mu_{0} H^{2}\right)$ is the Mason number proportional to the ratio of the hydrodynamic-tomagnetic forces acting on the aggregates. Note that the Mason number could also be defined through either suspension magnetization or particle magnetization, $M$, instead of the magnetic field $H$ [Klingenberg et al. (2007)]. The definition $M a(M)$ allows a better representation of experimental results in the broad range of the magnetic fields. However, in the present case of the linear magnetization law, $M \propto H$, the definition $M a(H)$ is equivalent to the Klingenberg's definition $M a(M)$ and seems to be more convenient for direct comparison with experiments because it does not require additional measurements of the suspension magnetic susceptibility. 
The drops do not flip under the shear flow until a critical Mason number, $M a_{c}$, which can be estimated on the basis of the more general approach of Okagawa et al. (1974) who have considered the motion of an ellipsoidal particle under shear and electric fields. This theory gives $M a_{c}=3 \chi^{2} \ln r /(8 r(2+\chi))$, whose value is estimated to be of the order of unity in our model conditions. Since our theory is applied to extremely low Mason numbers, the non-flipping condition, $M a<M a_{c}$ is automatically satisfied.

The aggregate volume $V$ and aspect ratio $r$ are defined by a condition of mechanical stability of the aggregates. It is clear that the hydrodynamic forces tend to break the aggregates, while the magnetic and the surface ones tend to keep them intact. Two possible mechanisms are expected to govern this force balance. On the one hand, the whole aggregate may become unstable at some critical volume, $V_{c}$, when the tensile force acting on the aggregate is not counterbalanced any more by the compressive force. On the other hand, the aggregate growth is limited by the balance between the erosive hydrodynamic and adhesive magnetic forces acting on particles situated on the aggregate surface. Both mechanisms lead to the aggregate destruction. The former one will be hereinafter called the "bulk destruction mechanism", while the latter one will be referred to as the "erosive destruction mechanism". These two mechanisms are considered in details in the next two subsections, where the aggregate aspect ratio and volume are estimated.

\section{B. Force equilibrium: bulk destruction mechanism}

We will take into account that the angle $\theta$ for the highly elongated drops is small (it will be justified below), that is why we will use the estimate: $\tan \theta \approx \sin \theta \approx \theta$, and $\cos \theta \approx 1$. In the frame of the bulk destruction mechanism, we will ignore the behavior of the magnetic particles on the aggregate surface. The balance between the stretching and contracting forces will therefore be applied to the whole aggregate. The total force $f$, which tends to deform the drop, consists of the hydrodynamic force $f_{h}$ and the potential force $f_{p}$. In its turn, the last force consists 
of the force $f_{m}$ of the demagnetizing field of the drop (this force, like $f_{h}$, tends to elongate the drop) and the force $f_{\sigma}$ of the surface tension, which tends to transform the drop into a sphere.

The hydrodynamic force can be deduced from Batchelor's slender body theory [Batchelor (1970)], as:

$$
f_{h} \approx \frac{\pi a^{2}}{\ln r} \eta_{0} \dot{\gamma} \sin \theta \cos \theta \approx \frac{\pi a^{2}}{\ln r} \eta_{0} \dot{\gamma} \theta
$$

Here $a$ is the major semi-axis of the drop. For the further calculations it will be convenient to express $a$ through the drop volume $V$ and the aspect ratio $r$. One can easily show that, for the ellipsoidal drop, these three magnitudes are related by the following formula:

$$
a=\left(\frac{3 V}{4 \pi}\right)^{1 / 3} r^{2 / 3}
$$

Substituting (5) into (4), we get:

$$
f_{h} \approx \pi\left(\frac{3 V}{4 \pi}\right)^{2 / 3} \frac{r^{4 / 3}}{\ln r} \eta_{0} \dot{\gamma} \theta
$$

The potential force $f_{p}$ can be calculated using the following relation:

$$
f_{p}=-\left.\frac{\partial F}{\partial l}\right|_{V}=-\left.\frac{1}{2} \frac{\partial F}{\partial a}\right|_{V}=-\left.\frac{1}{2} \frac{\partial F}{\partial r} \frac{\partial r}{\partial a}\right|_{V}
$$

Here $F$ is the drop free energy, $l=2 a$ is the length of the drop major axis. The free energy can be presented as:

$$
F=F_{m}+F_{\sigma}
$$

where $F_{m}$ is the free energy of the drop interaction with the field $H, F_{\sigma}$ is the free energy of the drop surface tension.

In the framework of the approximation $\theta<<1, r>>1$, we get the following expressions for the magnetic and surface energies (see, for example, Landau and Lifshitz (1960); Zubarev and Ivanov (1998)):

$$
F_{m}=-\frac{\mu_{0}}{2} V H^{2} \frac{\chi}{1+\chi r^{-2} \ln r}
$$


$F_{\sigma}=\sigma_{s} S \approx \sigma_{s} \pi^{2}\left(\frac{3}{4 \pi}\right)^{2 / 3} V^{2 / 3} r^{1 / 3}$

Here $\sigma_{s}$ is the coefficient of the surface tension, $S$ is the surface area of an elongated ellipsoid with volume $V$ and aspect ratio $r$. The well known form of $S$ as function of $V$ and $r$ (see, for example, Korn and Korn (1968)) is used here in the limiting case $r>>1$.

The surface tension coefficient is typically estimated as the difference between the energy of interactions between neighboring particles in the bulk of the aggregate and on the aggregate surface per unit surface [Cutillas et al. (1998), Iskakova et al., (2006)]. Since we consider only magnetic interactions between particles, the interaction energy scales as $\mu_{0} M^{2} R^{3}$ which gives the following scaling for the surface tension:

$$
\sigma_{s} \propto \mu_{0} M^{2} R=\mu_{0} \chi^{2} H^{2} R
$$

where $R$ is a length-scale of the order of magnitude of the particle size and $\chi_{p}$ is the particle magnetic susceptibility. In a real situation, the surface tension appears to depend on the orientation of the magnetic field with respect to the aggregate surface [Lobkovski and Halsey (1995), Cebers (2002)]. Such anisotropy leads to surface instability in the case of concentrated ferrofluid droplets, which appear as a result of phase separation in a magnetic colloid [Promislow and Gast (1997)]. We expect that these effects do not change the qualitative behavior of MRS and we neglect them at the present approximation.

Combining Eqs. (4)-(11), after calculations one can get the following expression for the total tensile force acting on the drop:

$$
\begin{aligned}
& f=V^{2 / 3}\left[\alpha \mu_{0} H^{2} r^{-8 / 3} \ln r+\pi\left(\frac{3}{4 \pi}\right)^{2 / 3} \frac{r^{4 / 3}}{\ln r} \eta_{0} \dot{\gamma} \theta\right]-V^{1 / 3} \beta \mu_{0} H^{2} r^{-1 / 3} \\
& \text { where } \alpha=\frac{3}{4}\left(\frac{4 \pi}{3}\right)^{1 / 3} \chi^{2} \text {, and } \beta=\frac{\pi^{2}}{4}\left(\frac{3}{4 \pi}\right)^{1 / 3} \chi^{2} R .
\end{aligned}
$$

Schematically the dependence of the force $f$ on the aspect ratio $r$ is shown in Fig.2. 


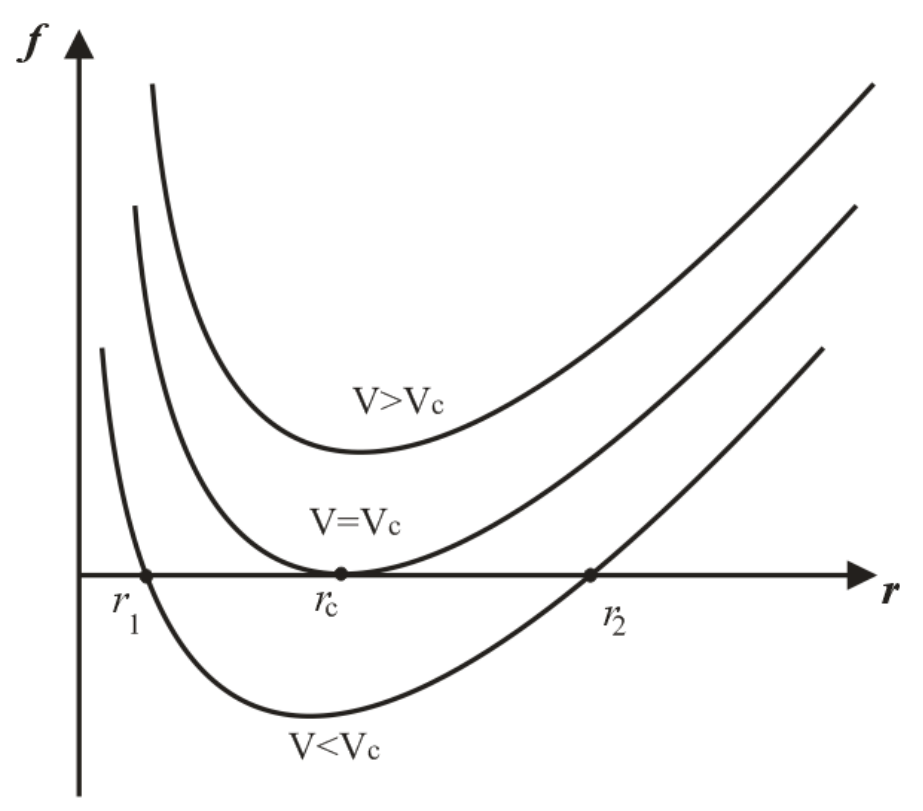

Fig.2. The tensile force $f$ (in arbitrary units) vs. the drop aspect ratio $r$. The meaning of parameters $\mathrm{V}_{\mathrm{c}}$ and $\mathrm{r}_{\mathrm{c}}$ is explained in the text.

The negative part of $f$ corresponds to contraction of the drop, positive ones - to its elongation. The points $r_{1}$ and $r_{2}$ are stationary (with respect to the drop elongation) magnitudes of the aspect ratio $r$ of a drop with a given volume $V$. The ratio $r_{1}$ corresponds to a stable point of the drop deformation, $r_{2}$ - to an unstable point. If $V<V_{c}$, the drop has a stable aspect ratio $r_{1}$ and can grow due to the adsorption of single particles, amalgamation with other drops, etc. If the volume $V$ is larger than the volume $V_{c}$, the force $f$ is positive for all the values of $r$. This means that the elongating forces dominate the surface tension force and one can expect the stretching of the drop till its break somewhere near the drop middle plane. The growth of the drop volume will stop when $V$ achieves $V_{c}$. Thus, the volume $V_{c}$ and the aspect ratio $r_{c}$ of the stable drop are determined as a solution of the system of equations:

$$
f(V, r)=0,\left.\frac{\partial f(V, r)}{\partial r}\right|_{V}=0
$$

To close the problem, one should take into account the dependence (3) of the angle $\theta$ on the aspect ratio $r$, such that the system (13) takes the final form as follows: 


$$
\begin{aligned}
& V^{2 / 3}\left[\alpha \mu_{0} H^{2} r^{-8 / 3} \ln r+M a \frac{2(2+\chi)}{\chi^{2}} \pi\left(\frac{3}{4 \pi}\right)^{2 / 3} \frac{r^{10 / 3}}{\ln ^{2} r} \eta_{0} \dot{\gamma}\right]-V^{1 / 3} \beta \mu_{0} H^{2} r^{-1 / 3}=0 \\
& V^{2 / 3}\left[-\frac{8}{3} \alpha \mu_{0} H^{2} r^{-1 / 3} \ln r+\frac{10}{3} M a \frac{2(2+\chi)}{\chi^{2}} \pi\left(\frac{3}{4 \pi}\right)^{2 / 3} \frac{r^{7 / 3}}{\ln ^{2} r} \eta_{0} \dot{\gamma}\right]+\frac{1}{3} V^{1 / 3} \beta \mu_{0} H^{2} r^{-4 / 3}=0
\end{aligned}
$$

After simple calculations we get the following solution for the set $(r, \theta, V)$ of unknown parameters characterizing the suspension microstructure:

$$
\begin{aligned}
& \frac{r^{2}}{\ln r}=\kappa \cdot M a^{-2 / 3} \\
& \theta=\frac{7 \chi^{2}}{11 \kappa^{2}} M a^{1 / 3} \\
& V=\left(\frac{11 \beta}{18 \alpha}\right)^{3} \frac{r^{7}}{\ln ^{3} r}=\left(\frac{3}{4 \pi}\right)^{2}\left(\frac{11 \pi^{2} R}{54}\right)^{3} \frac{r^{7}}{\ln ^{3} r}
\end{aligned}
$$

with $\kappa=\left[\frac{7 \alpha}{22 \pi}\left(\frac{4 \pi}{3}\right)^{2 / 3} \frac{\chi^{2}}{(2+\chi)}\right]^{1 / 3}=\left[\frac{7 \chi^{4}}{22(2+\chi)}\right]^{1 / 3}$ being a numerical factor depending on the aggregate magnetic susceptibility $\chi$. Eq. (15b) is obtained from Eq. (3) by replacing $r^{2} / \ln r$ by Eq. (15a). For brevity we omit in Eqs. (15) the subscript "c" in the aspect ratio $r$ and volume $V$ of the stable drop.

One can see from Eqs. (15a) and (15b) that our input assumptions, concerning the large aspect ratios, $r>>1$, and the small angles, $\theta<<1$, are justified in the limit of low Mason numbers, $M a<<1$.

\section{Force balance: erosive destruction mechanism}

We will now ignore the stability of the whole drop and consider the stability of the particles situated on the aggregate surface. Note that the erosive hydrodynamic force is maximal near the aggregate extremity situated on its major axis. Let us estimate the balance of hydrodynamic and magnetic forces at this point. In the considered limit, $M a<<1, r>>1$, and, 
$\theta<1$, the expressions for these forces reduce to the following ones (details of derivation are provided in the Appendices I and II):

$$
\begin{aligned}
& f_{h} \approx \eta_{0} \dot{\gamma} \frac{r^{2}}{\ln r} \theta \cdot 4 \pi R^{2} \\
& f_{m} \approx-\frac{8}{3} \pi \delta \chi(1+\chi) r^{2} \frac{\mu_{0} H^{2} R^{3}}{a}
\end{aligned}
$$

with $\delta=3 \chi_{p} /\left(3+\chi_{p}\right)$ and $\chi_{p}$ being the particle magnetic susceptibility. The force balance reads:

$$
f_{h}+f_{m}=0
$$

In conventional MR suspensions subjected to low-to-moderate magnetic fields, the particle magnetic susceptibility is of the order of $\chi_{p} \sim 10^{2}$, which results in $\delta \approx 3$.

The torque balance (3) and the force balance (16c) do not still give a closed system of equations for the desired quantities $\theta$ and $r$ because an unknown parameter, the length of the aggregate major semi-axis, $a$, appears in Eq. (16b) for the magnetic force. This magnitude is related to the aggregate volume, $V$, by Eq. (5). Strictly speaking, the aggregate volume depends on the kinetics of the nucleation/aggregation process, which is influenced by the flow. However, the characteristic time scale of amalgamation and disintegration of the aggregates, as well as the time scale of the change of the aggregate shape in response to its volume variation, appears to be much shorter than the typical time, $\dot{\gamma}^{-1} \Phi^{-1 / 3}$ of the mutual approach of two aggregates - the time required for an aggregate to move a distance equal to the mean space between neighboring aggregates. Such a difference in time scales is expected in the considered case of low Mason numbers and low particle volume fractions. Thus, ignoring the effect of the tensile hydrodynamic forces, one can determine the aggregate aspect ratio $r$, at a given aggregate volume $V$, from the condition of minimum of the drop free energy $F$ with respect to $r$. Of course, this condition is equivalent to the equation $f_{p}=0$, where the potential tensile force $f_{p}$ is determined by Eq.(7) in the frame of the bulk destruction model. 
For highly elongated aggregates the equation $(\partial F / \partial r)_{V, \theta}=0$ takes the form [Zubarev and Ivanov (1998)]:

$V=\frac{\pi^{4} R^{3}}{48} \frac{r^{7}}{\ln ^{3} r}$

Relationship (17) has been obtained for an aggregate parallel to the magnetic field. When the Mason number is small, the angle $\theta$ between the aggregate major axis and the magnetic field direction is small. Therefore Eq. (17) can be used as a first approximation in the low Mason number limit. Now, applying the torque balance [Eq. (3)] and the force balance [Eqs. (16a)(16c)], eliminating the aggregate major semi-axis $a$ with the help of Eqs. (5), (17), we arrive at the following transcendent equation for the aggregate aspect ratio:

$\frac{r^{5 / 2}}{\ln ^{3 / 2} r}=\vartheta M a^{-1}$

where $\vartheta=\left(\frac{4 \delta \chi^{3}(1+\chi)}{3 \pi(2+\chi)}\right)^{1 / 2}$.

Equation (18) demonstrates that the drop is highly elongated ( $r>>1$ ) when the inequality $M a<<1$ is held. In order to find an approximate analytical expression for the aspect ratio $r$, let us calculate the logarithms of both sides of Eq. (18). Neglecting the term $\ln (\ln r)$ as compared with $\ln r$, we get:

$$
\ln r \approx \frac{2}{5} \ln \left(\vartheta M a^{-1}\right)
$$

This allows us to obtain the final relationships for the set $(r, \theta)$ of microstructure parameters:

$$
\begin{aligned}
& r \approx\left(\frac{2}{5}\right)^{3 / 5} \vartheta^{2 / 5} M a^{-2 / 5} \ln ^{3 / 5}\left(\vartheta M a^{-1}\right) \\
& \theta \approx\left(\frac{5}{2}\right)^{2 / 5} \frac{2(2+\chi)}{\chi^{2}} \vartheta^{4 / 5} M a^{1 / 5} \ln ^{1 / 5}\left(\vartheta M a^{-1}\right)
\end{aligned}
$$

As it is seen from Eqs. (20a), (20b), the approximations $r>>1, \theta<<1$ are justified for the limit of low Mason numbers, $M a<<1$, in a similar way as in the "bulk destruction" model. 


\section{Shear stress and field-specific viscosity}

The stress tensor generated by the drops can be presented as the sum of a symmetric part (stresslet) and an asymmetric part (coming from the external torque). Applying the well-known expression [Brenner (1974), Pokrovskiy (1978)] for the stress tensor generated by anisotropic particles to the case of highly elongated drops ( $r>>1)$, we get the following formula for the shear component of the aggregate stress:

$$
\sigma_{a}=\sigma_{\text {symmetric }}+\sigma_{\text {asymmetric }}=\left[\frac{2}{3} \Phi \eta_{0} \dot{\gamma} \frac{r^{2}}{\ln r} \cos ^{2} \theta \sin ^{2} \theta+\frac{1}{2} n_{a} \Gamma_{h} \cos (2 \theta)\right]+\frac{1}{2} n_{a} \Gamma_{h},
$$

where $\Gamma_{h}$ is the hydrodynamic torque, acting on the drop, $n_{a}=\Phi / V$ is the number of drops in a unit volume of the system. In the limit of small angles $\theta$, the first term in the brackets of Eq. (21) becomes negligible and both the symmetric and the asymmetric parts give equal contributions to the total stress. Taking into account the expression for $\Gamma_{h}$ [right-hand side of Eq. (2)] in the limit $\theta<1$, equation (21) reduces to:

$$
\sigma_{a} \approx \frac{\Phi}{V} \Gamma_{h}=2 \Phi \eta_{0} \dot{\gamma} \frac{r^{2}}{\ln r}
$$

Substituting Eq. (15a) or Eqs. (19), (20a) into (22), we get the two expressions for the aggregate stress, within the frames of the bulk destruction, $\sigma_{a, b u l k}$, or erosive destruction, $\sigma_{a, \text { erosive }}$, models:

$$
\begin{aligned}
& \sigma_{a, \text { bulk }}=2 \kappa \Phi\left(\eta_{0} \dot{\gamma}\right) M a^{-2 / 3}=2 \kappa \Phi\left(\mu_{0} H^{2}\right)^{2 / 3}\left(\eta_{0} \dot{\gamma}\right)^{1 / 3} \\
& \sigma_{a, \text { rossive }}=2\left(\frac{2}{5}\right)^{1 / 5} \Phi\left(\eta_{0} \dot{\gamma}\right) \vartheta^{4 / 5} M a^{-4 / 5} \ln ^{1 / 5}\left(\Im M a^{-1}\right) \propto \Phi\left(\eta_{0} \dot{\gamma}\right)^{1 / 5}\left(\mu_{0} H^{2}\right)^{4 / 5}
\end{aligned}
$$

Using the definition of the field-specific viscosity of the suspension $[\eta]=\left(\sigma-\eta_{0} \dot{\gamma}\right) /\left(\varphi_{0} \eta_{0} \dot{\gamma}\right)=\sigma_{a} /\left(\varphi_{0} \eta_{0} \dot{\gamma}\right)$, we get the following expression for this magnitude in the frame of both models:

$$
[\eta]_{b u l k}=2 \frac{\kappa}{\varphi_{a}} \cdot M a^{-2 / 3}
$$


$[\eta]_{\text {erosive }}=\frac{2}{\varphi_{a}}\left(\frac{2}{5}\right)^{1 / 5} \vartheta^{4 / 5} M a^{-4 / 5} \ln ^{1 / 5}\left(\vartheta M a^{-1}\right)$

Recall that $\varphi_{a} \approx 0.64$ is the internal volume fraction of the aggregates. The relationships (22) and (23) represent the main results of our theory.

It is interesting to note that, for both models, the aspect ratio (15a) or (20a), and, therefore, the stress $(22 a, b)$ and the field-specific viscosity (23a,b), do not depend on the particle radius $R$ intervening into the surface tension (11) of the drop. This result can be explained from the scaling arguments. In more details, the particle size is the only dimensional scale of the problem in the unbounded non-Brownian suspension with colloidal interactions negligible as compared to the magnetic ones. Therefore, this parameter cannot form any dimensionless group and automatically works out from the dimensionless magnitudes $r$ and $[\eta]$. Consequently, it does not appear in the aggregate stress. On the contrary, the aggregate stable volume $V$ (the dimensional quantity) scales as $R^{3}$ as inferred from Eqs. (15c), (17).

Recall that the upper bound, $M a_{u p}$, of the Mason number for both considered models corresponds to the aggregate aspect ratio $r \approx 10$. Above $M a_{u p}$ approximations $r>>1$ and $\theta<1$ do not hold. This gives $M a_{u p} \approx 0.1$ for the bulk destruction model and $M a_{u p} \approx 0.35$ for the erosive destruction model. On the other hand, at very low Mason numbers, the structures can span the gap of the flowing channel and the aggregate behavior will be strongly affected by the interactions with the walls. The lower bound of Mason number, $M a_{l o w}$ is therefore defined by limiting the aggregate length $2 a$ by the gap $g$ between the walls. Applying an energy minimization analysis [described in details by Grasselli et al. (1994)], we estimate the aspect ratio of the ellipsoidal aggregates confined by two plains of the order of $r \sim 10^{2}$ for a typical gap-to-particle radius ratio, $g / R \sim 10^{3}$. This allows us to estimate the order of magnitude of the lower bound of our model by using Eqs. (15a) and (20a), namely $M a_{\text {low }} \sim 10^{-3}$. 
In what follows, in Sec. III-A, we analyze the theoretical results in comparison with the experimental ones for the range of Mason numbers, $M a_{\text {low }}<M a<M a_{\text {up }}$. Discussion on the rheological behavior beyond this limit will be presented in Sec.III-B.

\section{Results and discussion}

\section{A. Power-law behavior at $M a_{\text {low }}<M a<M a_{\text {up }}$}

First of all, let us remark the different rheological behavior predicted by both models. As inferred from Eqs. (23a,b), the two models predict different scaling of the field specific viscosity: the bulk destruction mechanism provides $[\eta]_{b u l k} \propto M a^{-2 / 3}$, while the erosive destruction mechanism gives a stronger decrease with Mason number, $[\eta]_{\text {erosive }} \propto M a^{-4 / 5}$ if we neglect the logarithmic term in Eq. (23b). Analysis shows that the former mechanism leads to smaller volumes and smaller aspect ratios of the aggregate as compared to the latter mechanism. It would be therefore logical to maintain the bulk destruction mechanism as the one defining the aggregate behavior and therefore the suspension rheology. However, most of the experimental data reported in literature (we will analyze some of these data below) give a behavior closer to that predicted by the erosive destruction model, i.e. $[\eta] \propto M a^{-4 / 5}$. It is possible that the aggregate extension under the action of the demagnetizing field (supposed in the bulk destruction model) is hindered by the solid friction between the particles constituting the aggregates. In this case, the aggregate could grow beyond the critical volume of the bulk destruction [Eq. (15c)] until the onset of the particle erosion from the aggregate surface, such that the erosion mechanism dominates. At this moment, we cannot give a clear response about which mechanism is dominant, or about whether both appear simultaneously. Therefore, we inspect the behavior of both models.

The dependency of the field-specific viscosity $[\eta]$ on the Mason number is presented in Fig.3, where our both models are compared with those of Halsey et al. (1992) and Shulman et al. 
(1984), as well as with three set of experimental data obtained by different research groups. Before inspecting this figure, we shall briefly describe how we plotted the theoretical and experimental data of other researches. The model of Halsey et al. (1992) gives the same expression as our equation (23a) for the field-specific viscosity, in which the numerical factor $\kappa$ is somewhat different from the one of our bulk destruction model and is equal to $\kappa=\left(\frac{\chi^{4}}{32(2+\chi)}\right)^{1 / 3}$ (note that this magnitude is not explicitly given in their paper). This parameter (and, consequently, the field-specific viscosity [ $\eta]$ ) appears to be about two times lower than the one of our model. The possible reason of this disagreement is that in the model of Halsey et al. (1992), the shape of the drop was determined from the only condition of minimum of the drop free energy, while the mechanisms of aggregate destruction by the hydrodynamic forces was not taken into account. In what concerns the model of Shulman et al. (1984), it gives another scaling for the field-specific viscosity, namely $[\eta]=\left(2 \kappa / \varphi_{a}\right) \cdot M a^{-1}$ with $\kappa=\frac{1+\chi}{6 \varphi_{a}} \frac{R}{\Delta}$ and $\Delta$ being the mean interparticle gap inside the aggregates, $R$ - the particle radius and $\varphi_{a} \approx 0.64-$ the internal volume fraction of the aggregates. All the four compared models are based on the assumption of ellipsoidal dense drop-like aggregates.

We remark that the results of these models depend on the magnetic susceptibility $\chi$ of the drop-like aggregates, which intervenes into the expressions for $\kappa$ and $\vartheta$ parameters. Simulations, based on the finite element method [described in details by López-López et al. (2012)], give the value $\chi \approx 12$ for the case of magnetic particles of high magnetic permeability. For definiteness, we use this value, $\chi=12$, for the four theoretical models to be compared. On the other hand, the Shulman's model uses an adjustable parameter $\Delta / R$, the gap-to-the particle size ratio, whose value is expected to be of the order of $10^{-2}$, but that must be forced to an incredibly large value $(\Delta / R=1)$ in order to adequately describe the experimental results. 
All the three sets of experimental data taken from literature, and providing a power law scaling, were fitted to the power-law dependency $[\eta] \propto M a^{-v}$, with $v$ being a real exponent. We also renormalized the Mason number used by different authors to the one used in our work, $M a=\eta_{0} \dot{\gamma} /\left(\mu_{0} H^{2}\right)$, taking into account that the magnetic contrast factor $\left(\mu_{p}-1\right) /\left(\mu_{p}+2\right)$ intervening into other definitions is close to unity for the case of particles of high magnetic permeability $\mu_{p}>>1$.

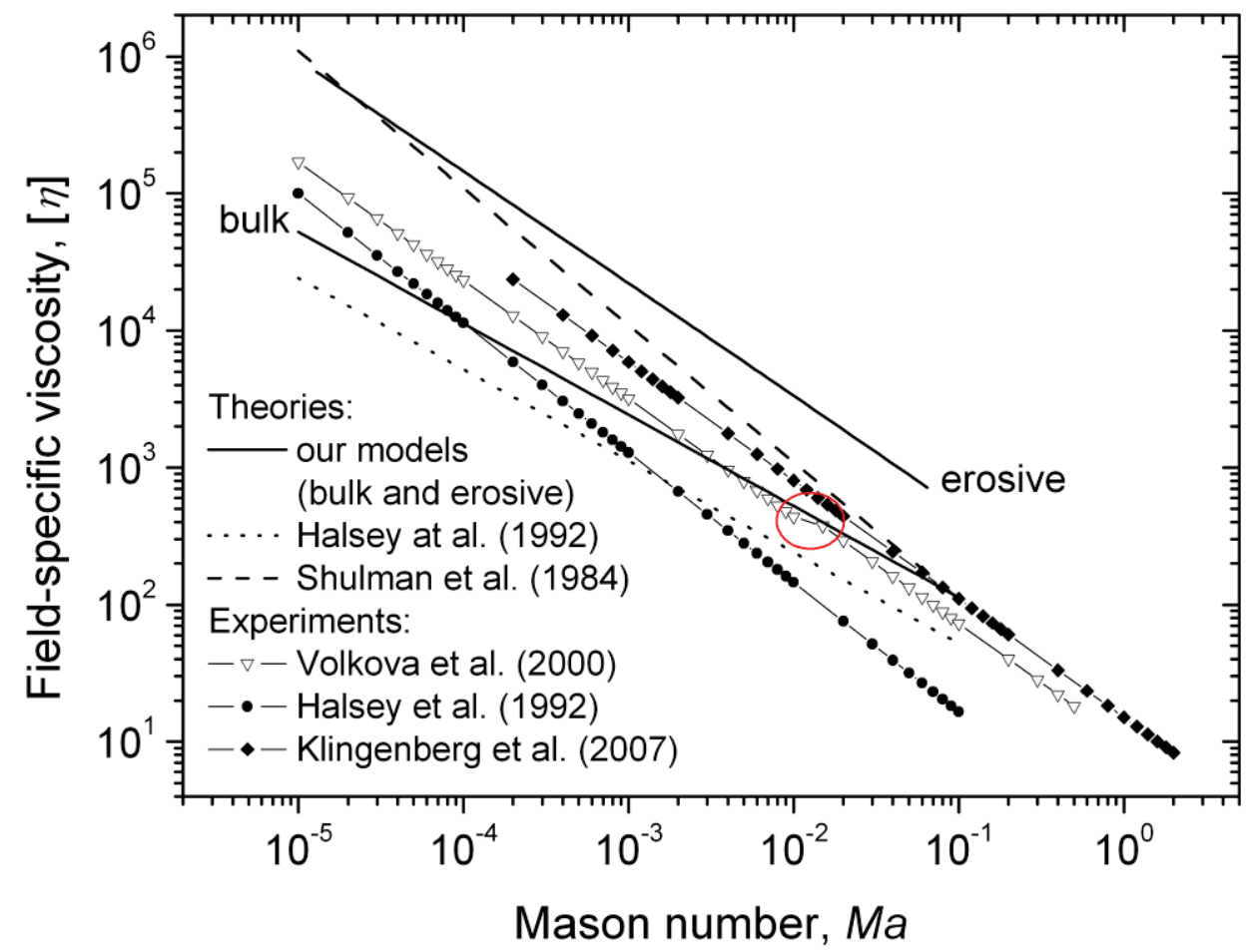

Fig.3. Theoretical and experimental dependencies of the field-specific viscosity vs. the Mason number. Experimental curves represent the best fit to the power law $[\eta] \propto M a^{-v}$ of the experimental data of Halsey et al. (1992) (electrorheological fluid composed of silica particles dispersed in 4-methylcyclohexanol at $10 \%$ vol and at the applied electric field of $1 \mathrm{kV} / \mathrm{m}$ ); Volkova et al. (2000) (MRS composed of carbonyl iron particles dispersed in a silicon oil at $5 \% \mathrm{vol}$ and at $7<H<20 \mathrm{kA} / \mathrm{m}$, a red circle indicates the position of a small "shoulder" of the flow curve); Klingenberg et al. (2007) (MRS composed of iron particles in hydrocarbon oil at $\varphi=45 \%$ vol, we had to renormalize these data to the linear magnetization limit used in the three compared theories, such that the proportionally factor between our and Klingenberg's et al. definitions of the Mason number is $2 \chi_{s}{ }^{2} /(9 \varphi)$, with the value $\chi_{s} \approx 4.2$ of the suspension magnetic susceptibility found form the interpolation formula proposed by the same authors for the suspension magnetization in the limit of vanishing fields)

Thus, inspecting figure 3, we see that all the three set of experimental data give nearly the same power-law trend with an exponent $v=0.85-0.9$ lying between 2/3, predicted by the Halsey's et al. model, and 1, predicted by the Shulman's et al. model. Despite the quantitative difference, our erosive destruction model gives a better qualitative agreement with experiments, giving an 
exponent $v=0.8$. The discrepancy between the inspected models and the experimental results is likely due to the fact that none of the two rupture mechanisms (proposed by our both models) appears solely but it is rather their synergy that may provide the correct scaling. Quantitatively, the experimental results obtained by different groups are quite different and unfortunately do not gather into the same master curve. This complicates the comparison between theory and experiments. For instance, the model of Shulman et al. (1984) gives a better correspondence with the results of Klingenberg et al. (2007) on highly concentrated MRS (at the expense of an abnormally high interparticle gap parameter $\Delta / R=1$ ) and our bulk destruction model- with the results of Volkova et al. (2000) on MRS composed of highly permeable carbonyl iron particles. Nevertheless, recall that our bulk destruction theory employs a new alternative mechanism of the aggregate destruction. As already mentioned, combined with the classical mechanism of the particle rupture from the aggregate surface (erosive destruction), we expect to recover the intermediate behavior with an exponent $2 / 3<v<1$ closer to experimental values and to get a better quantitative agreement with the experimental results.

Another point that can explain the scatter of the experimental results is the solid friction between the particles, since this solid friction can prevent the aggregates from deformation accordingly to the minimum of the free energy. An estimation of the friction force between two iron particles is obtained from the radial magnetic force times a friction coefficient, which is usually between 0.2 and 0.3 . As a typical example, the experimental radial magnetic stress $\left(F_{m} / a^{2}\right.$ ) between two iron particles for a field $H=24 \mathrm{kA} / \mathrm{m}$ is $100 \mathrm{kPa}$ (Volkova et al. 2002), which gives a frictional stress of $20 \mathrm{kPa}$ on the particles located on the surface of the drop. This stress is far from being negligible and will likely prevent the drop from reaching its equilibrium volume [cf. Eqs. (15c) and (17)] in both cases of bulk or erosive destruction. 


\section{B. Beyond the limit $M a_{\text {low }}<M a<M a_{\text {up }}$}

A deviation of the experimental results from a simple power-law trend at very low or relatively high Mason numbers has been reported by some groups. As an example, we plot in Fig. 4. the experimental dependencies of the field-specific viscosity versus the Mason number obtained by Berli and de Vicente (2012) for suspensions of carbonyl iron particles, as well as our new experimental results obtained for suspensions of cobalt spheres (see details in figure caption). As we see, both sets of experimental results essentially coincide in the range $10^{-}$ ${ }^{4}<M a<10^{-2}$. As seen in the inset of Fig. 4 , in this range, the experimental data are perfectly fitted by a power law trend $[\eta] \propto M a^{-v}$ with the same exponent, $v=4 / 5$, as the one predicted by our erosion destruction model. At Mason numbers $M a>M a_{u p} \sim 0.1$ the data no longer follow the power-law trend and are not gathered into a master curve. Furthermore, the data of our experiments show an increasing slope at high $M a$, while those reported by Berli and de Vicente (2012) exhibit a decreasing slope. We think that at high Mason numbers, the role of field becomes weaker and, possibly, colloidal interactions between particles start playing a nonnegligible role. In this case suspension rheology is no longer governed solely by Mason number but also by an additional dimensionless number, like, for example, the ratio of colloidal-tomagnetic forces. The data no longer collapse into a single master curve. The divergence between Berli and de Vicente and our data at high Ma could come from a different colloidal stabilization of the magnetic particles (no stabilizing agents in the first study and surfactant stabilization in our study) leading to different colloidal interactions. 


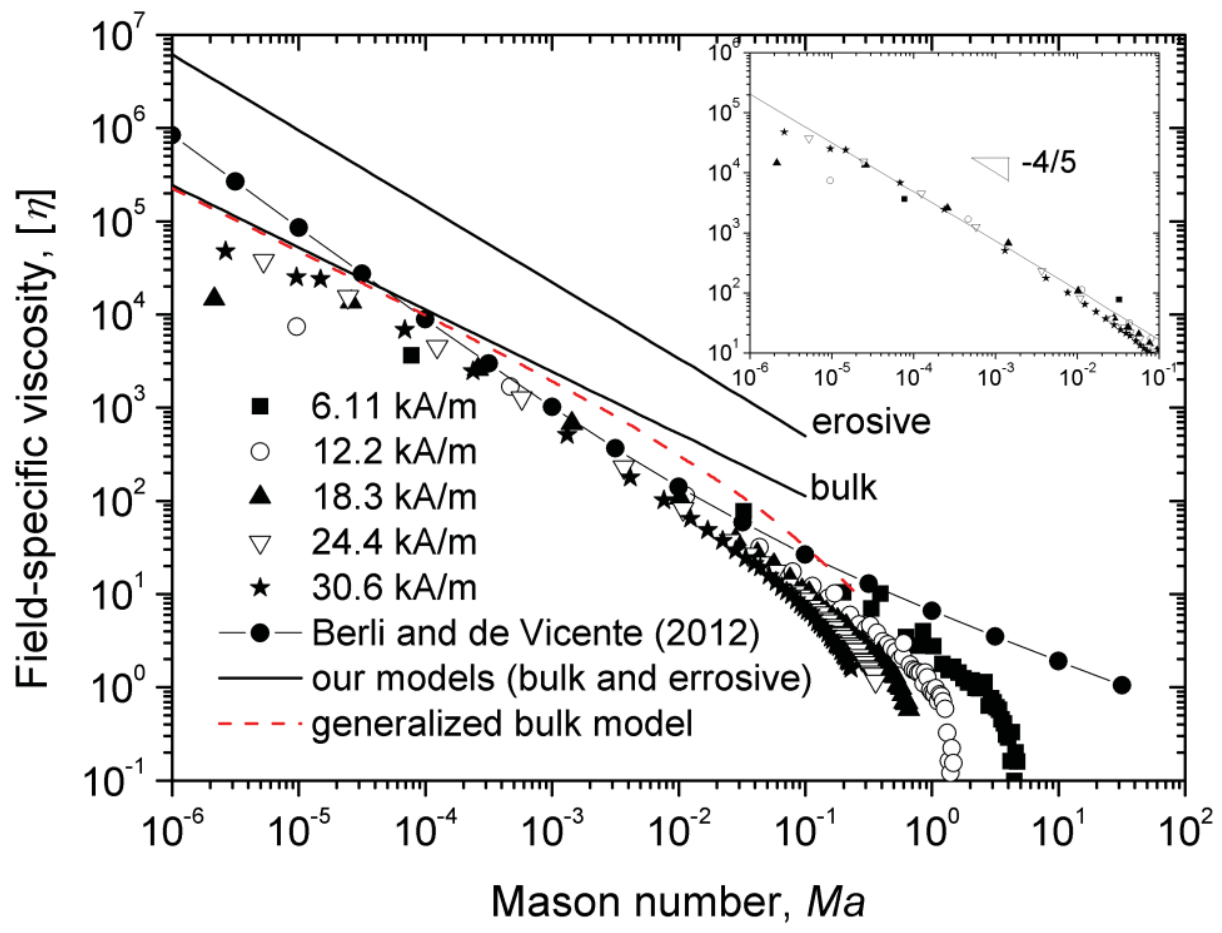

Fig.4. Mason number dependency of the field-specific viscosity in a broad range of Mason numbers. Experimental data of Berli and de Vicente (2012) (full circles connected by a solid line) were obtained for a 10\%vol suspension of carbonyl iron particles (diameter $800 \mathrm{~nm}$ ) dispersed in silicon oil without a stabilizing agent. According to Berli and de Vicente (2012), these data are perfectly fitted by Casson rheological equation written in the following form in terms of the field-specific viscosity: $[\eta]=\left(M a / M a^{*}\right)^{-1}+\left(M a / M a^{*}\right)^{-1 / 2}$, with $M a^{*}$ being an adjustable parameter. Our experimental results (symbols not connected by a line) were obtained for 5\%vol. suspensions of cobalt spherical particles (diameter $1.34 \mu \mathrm{m}$ ) dispersed in silicon oil and stabilized by adsorption of aluminum stearate on particle surface. These data are regrouped onto a single master curve in the range of Mason numbers, $10^{-4}<\mathrm{Ma}<10^{-2}$; outside this range, the data deviate from the master curve. Solid lines stand for the predictions of the bulk destruction [Eq. (23a)] and erosive destruction [Eq. (23b)] models in the limit of low Mason numbers. Red dashed curve corresponds to extension of the bulk destruction model to higher Mason numbers

In order to check the behavior at high Mason number, we have extended our bulk destruction model [Sec. II-B] beyond the limit $r>>1, \theta<<1$ and $M a<<1$. We present the corresponding Mason number dependency in Fig. 4 by a dashed line (mathematical details of this model are described in López-López et al. (2014)).

As we see, this generalized model (not accounting for colloidal interactions) gives a more rapid viscosity decrease with Mason number than that predicted by the bulk destruction model limited to low Mason numbers. The curvature of the generalized dependency appears to be qualitatively similar to that of our experimental curves, even though they diverge from the 
master curve at high values of $M a$. The solution is obtained for Mason numbers, $M a<0.25$ - the case when the magnetic torque still prevents the aggregates from flipping under shear flow.

At Mason numbers $M a<10^{-4}$, thus beyond the estimated lower bound, $M a_{\text {low }} \sim 10^{-3}$, the data are no longer gathered into a master curve and show different exponents ranging from $v \approx 0.5$ for some of our data to $v \approx 1$ for Berli and de Vicente experiments. As already stated, at $M a<M a_{\text {low }}$, wall effects come into play. These interactions may lead to qualitatively different behaviors. For a strong cohesion with the walls a static yield stress is usually observed and $M a^{-1}$ behavior is expected, as in the case of Berli and de Vicente experiments. For the opposite limit of perfect slippage of aggregates over the walls, a low-shear plateau (corresponding to $v=0$ ) may be observed, as predicted by [Gomez-Ramirez at al. (2011), cf. Eq. (24) in that paper] for suspensions of magnetic fibers. As inferred from Fig. 4, our experimental data show an intermediate behavior between perfect slippage and perfect adhesion. Furthermore, stick-slip instability at low shear rates may also be observed in some cases [López-López et al. (2013)]. In summary, diversity of the wall interaction scenarios does not allow, at the present time, a generalized description of the MR effect at Mason numbers $M a<M a_{\text {low }}$.

Finally, note that in the present models; we assumed that the extension of the drops by tensile hydrodynamic forces did not modify the gaps between the particles. This situation corresponds to a rather slow flow in the limit of low Mason numbers, $M a<<1$. At this limit, the particles inside the drops have enough time to rearrange in such a way to keep their contacts with neighboring particles. This provides the highest magnetic susceptibility and the lowest magnetic energy for a drop of a given aspect ratio and volume. At more rapid flows, the particles would not have enough time for such "ideal" rearrangement preserving interparticle contacts. Thus, the gaps between particles can be progressively "opened" during the drop extension. In this case, the drop magnetic susceptibility $\chi$ is expected to decrease with its elongation. The derivation of the magnetic energy (9) with respect to the drop length $l$ would result in an additional "compressive" term of the magnetic force, related to $\partial \chi / \partial l$ [López-López et al. (2012)]. This term can be quite 
important for the short-ranged magnetic interactions between particles of high magnetic susceptibility. In our previous work [Gomez-Ramirez et al. (2011)], we considered the opposite limit - we neglected the drop elongation but took into account the magnetic attraction between particles when the aggregate is ruptured at the middle and the gaps between particles are "opened". This led us to a balance between the "compressive" magnetic and tensile hydrodynamic forces and resulted in a Bingham behavior if the surface tension was neglected. In reality, there can be a combined effect of the four forces (the tensile and compressive magnetic forces as well as the surface tension and the hydrodynamic forces), which could lead to an intermediate behavior between $\sigma_{a} \propto \dot{\gamma}^{1 / 3}$ (or $[\eta] \propto M a^{-2 / 3}$ ) and the Bingham one ( $\sigma_{a}$ independent of $\dot{\gamma}$ and $[\eta] \propto M a^{-1}$ ). This study will be the subject of a separate work.

\section{Conclusions}

We present a model of magnetoviscous effect in magnetorheological suspensions with dense drop-like aggregates. Two different mechanisms of aggregate destruction by hydrodynamic forces are considered. Firstly, the aggregate may become mechanically unstable if the tensile hydrodynamic and tensile magnetic (coming from the demagnetizing field) forces overcome the compressive surface tension force. In this case, the aggregate is expected to break into parts and the aggregate rupture can be seen as a bulk destruction process. Alternatively, the magnetic particles can be eroded from the aggregate surface by the hydrodynamic forces; when these erosive forces overcome the adhesive magnetic ones, the aggregate size decreases until the equilibrium is reestablished. Such a scenario is referred to as erosion destruction mechanism. For a better understanding, we have considered both above-mentioned mechanisms separately, keeping in mind that they may act simultaneously, especially at higher suspension concentrations, when the aggregates may easily amalgamate.

Both mechanisms give qualitatively different rheological behaviors under the considered limit of Mason numbers, $M a_{\text {low }}<M a<M a_{\text {up }}$, where the lower bound, $M a_{\text {low }}$, corresponds to the 
percolation limit and the upper bound, $M a_{u p}$, - to the aggregate aspect ratio $r \approx 10$. The bulk destruction mechanism reproduces the power law $[\eta] \propto M a^{-2 / 3}$ of Halsey et al. (1992), even though it accounts for the hydrodynamic interaction in an essentially different way. The erosive destruction scenario provides another scaling for the field-specific viscosity, $[\eta] \propto M a^{-4 / 5}$, closer to the known experimental data. At the present moment, we are unable to give a grounded preference to the first mechanism or the second one of aggregate destruction, although experiments point out to the realization of the second one. Beyond the limit $M a_{\text {low }}<M a<M a_{u p}$, experimental data no longer follow a simple power law trend and do not gather into a single master curve. Diversity of wall interactions at $M a<M a_{\text {low }}$ and increasing importance of colloidal interactions at $M a>M a_{u p}$ may partially explain these behaviors.

In addition, analysis of the present model along with the theories available in literature reveals an extreme effect of the nature of the magnetic forces on the suspension rheological behavior. Depending on whether the magnetic force acting on the aggregates is tensile (due to demagnetizing fields, cf. Halsey et al. (1992) and the present bulk destruction model) or compressive (due to dipolar attractions between particles, cf. Shulman et al. (1984), GomezRamirez et al. (2011), also the erosive destruction model of the present study), the field-specific viscosity follows a power law $[\eta] \propto M a^{-v}$ with the exponent $v$ equal to either $2 / 3$ or $4 / 5$ or 1 . Most of the experimental results show an intermediate trend, $[\eta] \propto M a^{-v}$, with $v=0.7-0.95$. We believe that the synergy of both compressive and tensile magnetic forces will result in a correct rheological behavior with $2 / 3<v<1$ and will provide a better agreement with experiments. In this sense, the present model is the necessary step to the further development of the theory.

It is worth noting that the problem of definition of a leading contribution to the total magnetic force is not restricted to magnetic suspensions. In magnetic particle gels (ferrogels), the magnetic force leads to either contraction or extension of the sample along the applied magnetic 
field depending on the sample shape as well as on the internal particle space distribution [Stolbov et al. (2011), Zubarev (2012)].

For a longer perspective, the effects of magnetic and hydrodynamic interactions between particle aggregates should be carefully studied, especially for the case of concentrated MR suspensions. These interactions along with mutual collisions should unavoidably lead to stochastic destruction of the aggregates, followed by their reformation. The kinetics of the rupture-reformation events should considerably affect the average rheological properties of MR suspensions, even in steady flows. In summary, a rigorous approach combining a simultaneous determination of the aggregate orientation and the aggregate size distribution via the kinetics of the orientation-aggregation process is still missing in the domain of magnetorheology.

\section{Acknowledgments}

This work has been done under support of Russian Fund of Fundamental Investigations, grants 12-01-00132, 13-02-91052, 13-01-96047 and 14-08-00283; by the Act 211 Government of the Russian Federation № 02.A03.21.0006. The University of Granada (Acción Integrada con Rusia; Plan Propio 2011), as well as project CNRS PICS N 6102 are also acknowledged for their financial support.

\section{Appendix I. Demonstration of Eq. (16a) for the hydrodynamic force}

The hydrodynamic viscous force $\tau$, acting on the unit area of a rigid ellipsoid surface in a shear flow, have been calculated by Jeffrey (1922). In the case of aggregates with a high aspect ratio, $r>>1$, the components of these forces in the cylindrical reference frame $(\rho, z)$ (introduced in Fig.

1) read:

$$
\begin{aligned}
& \tau_{z}=\eta_{0} \dot{\gamma} P\left[\frac{8}{a b^{2}}\left(\frac{\sin 2 \theta}{24 \ln r} z a+\frac{2 \ln r \cos 2 \theta-1}{8 \ln r} \rho a\right)+\frac{\sin 2 \theta}{6 \ln r} \frac{a}{b^{2}}\right] \\
& \tau_{\rho}=\eta_{0} \dot{\gamma} P\left[\frac{8}{a b^{2}}\left(\frac{\cos 2 \theta+1}{4 \ln r} z a-\frac{\sin 2 \theta}{48 \ln r} r^{2} \rho a\right)+\frac{\sin 2 \theta}{6 \ln r} \frac{\rho}{b^{2}}\right]
\end{aligned}
$$


where $P=\left(\frac{z^{2}}{a^{4}}+\frac{\rho^{2}}{b^{4}}\right)^{-1 / 2}$.

The relations (A1) are valid in the shear plane formed by the flow velocity and velocity gradient. The normal component (with respect to ellipsoid surface) of this viscous force can be calculated as:

$$
\tau_{n}=\tau_{z} \frac{1}{\sqrt{1+s^{2}}}-\tau_{\rho} \frac{s}{\sqrt{1+s^{2}}}
$$

Here, the derivative $s=d z / d \rho=-\frac{a}{b^{2}} \frac{\rho}{\sqrt{1-\frac{\rho^{2}}{b^{2}}}}$ is determined on the ellipsoid surface.

Simple but cumbersome calculations show that, for all angles $\theta$ of our interest, the point where the normal force $\tau_{n}$ is maximal appears to be very close to the point $z=a, \rho=0$, i.e. to the extremity of the aggregate major axis. Combining Eqs. (A1)-(A2), we find the hydrodynamic force at this point:

$$
\tau_{n}(z=a, \rho=0)=\eta_{0} \dot{\gamma} \frac{r^{2}}{\ln r} \theta
$$

The hydrodynamic force, acting on a magnetic particle situated on the aggregate's extremity, can be estimated as follows:

$$
f_{h}=4 \pi R^{2} \tau_{n}(z=a, \rho=0)
$$

After substitution of Eq. (A3) into Eq. (A4), we arrive at the final result [Eq. (16a)] for the hydrodynamic force.

\section{Appendix II. Demonstration of Eq. (16b) for the hydrodynamic force}

The magnetic force acting on a particle situated on the aggregate extremity can be found from the following relation:

$$
f_{m}=\left.\frac{1}{2} \mu_{0} \mathrm{v}_{p} \delta \frac{\partial \aleph^{2}}{\partial z}\right|_{z=a, \rho=0}
$$


Here $\delta=\frac{3 \chi_{p}}{3+\chi_{p}}, \mathrm{v}_{p}=\frac{4}{3} \pi R^{3}$ is the particle volume, $\chi_{p}$ is the particle magnetic susceptibility, $\aleph$ is the absolute magnitude of the local magnetic field near the ellipsoid surface. In order to estimate the magnitude $\aleph$, let us present the external field $\mathbf{H}$ in the following form:

$\mathbf{H}=\mathbf{H}_{z}+\mathbf{H}_{\rho}, \quad H_{z}=H \cos \theta, \quad H_{\rho}=H \sin \theta \cos \psi$

Here $\psi$ is the polar angle in the cylindrical coordinate system. Under the approximation of the linear magnetization of the particles, we can consider separately the effects of the components $\mathbf{H}_{\mathrm{z}}$ and $\mathbf{H}_{\rho}$ on the force $f_{m}$. Let us introduce the scalar magnetic potential $\Psi$ according to the rule:

$\aleph=-\operatorname{grad} \Psi$

Following Landau and Lifshitz (1960), we will seek for the potential in the form

$$
\begin{aligned}
& \Psi=-H_{z} z\left(1+C \int_{\xi}^{\infty} q(\xi) d \xi\right), \\
& q(\xi)=\frac{1}{\left(\xi+a^{2}\right)^{3 / 2}\left(\xi+b^{2}\right)}
\end{aligned}
$$

Here $C$ is the integration constant. Relationships between ellipsoidal coordinates $\xi$ and $\zeta$ and cylindrical ones $z$ and $\rho$ can be found elsewhere [Landau and Lifshitz (1960)]. The ellipsoid surface corresponds to $\frac{z^{2}}{a^{2}}+\frac{\rho^{2}}{b^{2}}=1$, while its major axis $(\rho=0)$ is defined by $\zeta=-b^{2}$. The integration constant $C$ can be determined from the usual condition of continuity of the magnetostatic potential $\Psi$ across the aggregate surface. Computing this constant and deriving the magnetostatic potential according to Eq. (A7), we arrive at the following expression for the magnetic field intensity on the aggregate surface in the vicinity of the extremity $z=a, \rho=0$ :

$$
\begin{aligned}
& \aleph_{z}=H \cos \theta\left[1-\frac{\chi N}{(1+\chi N) J}\left(\int_{\xi}^{\infty} q(\xi) d \xi-z q(\xi) \frac{\partial \xi}{\partial z}\right)\right] \\
& \aleph_{\rho}=0
\end{aligned}
$$


where $\chi$ is the aggregate magnetic susceptibility, $J=\int_{0}^{\infty} q(\xi) d \xi$ and $N$ is the demagnetizing factor of the ellipsoid along its major axis. Substituting Eq. (A9) into Eq. (A5) and applying the approximation $r>>1, \theta<<1$, we arrive at the final expression (16b) for the magnetic force acting on a particle situated in the aggregate surface in the vicinity of its extremity.

\section{References}

Batchelor G.K., (a) “The stress system in a suspension of force-free particles” J.Fluid Mech. 41, 545-570 (1970)

Batchelor, G. K., (b) "Slender-body theory for particles of arbitrary cross-section in Stokes flow," J. Fluid Mech. 44, 419-440 (1970).

Baxter-Drayton Y. and J. F. Brady, « Brownian electrorheological fluids as a model for flocculated dispersions», J. Rheol. 40, 1027-1056 (1996)

Bayat N., Nethe A., Guldbakke J.M., Hesselbach J., Naletove V.A., Stahlmann H.-D., Uhlmann E., Zimmermann K., “Technical Applications” in Colloidal Magnetic Fluids, edited by S.Odenbach, (Springer, Berlin, 2009)

Berli C. L. A., and J. de Vicente, «A structural viscosity model for magnetorheology», Appl. Phys. Lett. 101, 021903 (2012)

Bonnecaze, R. T., and J. F. Brady, “Dynamic simulation of an electrorheological fluid,” J. Chem. Phys. 96, 2183-2202 (1992).

Bossis G., Volkova O, Lacis S., Meunier A., "Magnetorheology: Fluids, Structure and Rheology" in Ferrofluids, Magnetically Controllable Fluids and Their Application, edited by S. Odenbach (Springer, Berlin, 2002).

Bossis, G., E. Lemaire, O. Volkova, and H. Clercx, "Yield stress in magnetorheological and electrorheological fluids: A comparison between microscopic and macroscopic structural models," J. Rheol. 41, 687-704 (1997).

Brenner H., "Rheology of a dilute suspension of axisymmetric Brownian particles", Int. J. Multiphase Flow 1, 195-341 (1974).

Cebers A., "The anisotropy of the surface tension at the magnetic-field-induced phase transformations,” J. Magn. Magn. Mater. 252, 259-261 (2002)

Cutillas S, G Bossis, A Cebers «Flow-induced transition from cylindrical to layered patterns in magnetorheological suspensions » Phys. Rev. E 57, 804-811 (1998) 
de Gans B-J., H. Hoekstra and J. Mellema, « Non-linear magnetorheological behavior of an inverse ferrofluid," Faraday Discuss. 112, 209-224 (1999)

de Vicente J., D. J. Klingenberg and R. Hidalgo-Álvarez, "Magnetorheological Fluids: A Review”, Soft Matter, 7, 3701-3710 (2011)

Ginder J. M., "Behavior of magnetorheological fluids”, MRS Bull., 23 26-29 (1998).

Gomez-Ramirez A., P. Kuzhir, M. T. Lopez-Lopez, G. Bossis, A. Meunier, and J. D. G. Duran, "Steady shear flow of magnetic fiber suspensions: Theory and comparison with experiments", J.Rheol. 55, 43-67 (2011)

Grasselli, Y., G. Bossis, and E. Lemaire, "Structure induced in suspensions by a magnetic field," J. Phys. II 4, 253-263 (1994).

Halsey T., J. Martin, and D. Adolf, "Rheology of electrorheological fluids" Phys. Rev. Lett. 68, 1519-1523 (1992)

Iskakova L.Yu., Romanchuk A.P., Zubarev A.Yu. "Phase and structural transformations in magnetorheological suspensions”. Physica A., 366 18-30 (2006)

Jeffrey J.B., “The Motion of Ellipsoidal Particles Immersed in a Viscous Fluid”, Prog. Roy. Soc. A, 102, 161-179 (1922).

Klingenberg D.J., J.C. Ulicny and M.A. Golden, "Mason number for magnetorheology", J. Rheol. 51, 883-893 (2007)

Klingenberg, D. J., F. Vanswol, and C. F. Zukoski, “The small shear rate response of electrorheological suspensions. 1. Simulation in the point-dipole limit,” J. Chem. Phys. 94, 6160-6169 (1991).

Kordonsky V.I., Z.P. Shulman, S.R. Gorodkin, S.A. Demchuk, I.V.Prokhorov, E.A. Zaltsgendler, and B.M. Khusid, "Physical properties of magnetizable structure-reversible media”, J. Magn. Magn. Mater. 85, 114-120 (1990).

Korn G.A. and T.M. Korn «Mathematical Handbook for scientists and engineers”. McGrawHill Book Company, 1968

Landau L.D. and E.M. Lifshitz, Electrodynamics of Continuum Media, Pergamon Press, London, 1960

Liu J., E. M. Lawrence, A. Wu, M. L. Ivey, G. A. Flores, K. Javier, J. Bibette, and J. Richard., «Field-Induced Structures in Ferrofluid Emulsions”, Phys. Rev. Lett. 74, 2828-2831 (1995).

Lobkovsky, A. E. and Halsey, T.C. Surface energy anisotropy for dipolar lattices. J. Chem. Phys, 103, 3737 (1995) 
López-López M.T., P. Kuzhir, J. Caballero-Hernandez, L. Rodriguez-Arco, J.D.G. Durán, and G. Bossis. "Yield stress in magnetorheological suspensions near the limit of maximumpacking fraction.” J. Rheol. 56, 1209-1224 (2012).

López-López M.T., P. Kuzhir, L. Rodriguez-Arco, J. Caballero-Hernandez, J.D.G. Durán, and G. Bossis, "Stick-slip instabilities in the shear flow of magnetorheological suspensions." J. Rheol. 57, 1101-1119 (2013).

López-López M.T., P. Kuzhir and A. Zubarev, "Effect of drop-like aggregates on the viscous stress in magnetic suspensions", J. Non-Newt. Fluid. Mech. 208-209, 53-58 (2014).

Marshal L., C.F. Zukoski, and G.W.Goodwin "Effect of electric field on the rheology of non-aqueous concentrated suspensions”, J.Chem.Soc. Faraday Trans. 1, 85, 2785-2795 (1989)

Martin J.E., "Thermal chain model of electrorheology and magnetorheology” Phys.Rev.E. 63, 011406 (2000)

Martin J.E., and R. Anderson, “Chain model of electrorheology”, J.Chem.Phys. 104, 48144826 (1996)

Martin J.E., Venturini E., Gulley G.L and Williamson J. "Using triaxial magnetic fields to create high susceptibility particle composites”. Phys Rev. E, 69, 021508-1 -15 (2004).

Odenbach S., Magnetoviscous Effects in Ferrofluids (Springer, Berlin, 2002)

Okagawa, A., R. G. Cox, and S. G. Mason, "Particle behavior in shear and electric fields VI. The microrheology of rigid spheroids,” J. Colloid Interface Sci. 47, 536-567 (1974).

Pokrovskiy, V. N., Statistical Mechanics of Diluted Suspensions (Nauka, Moscow, 1978)

Promislow, J.H.E and Gast, A.P. Low-energy suspension structure of a magnetorheological fluid. Phys. Rev. E, 56, 642-651 (1997)

Ramos J., D. J. Klingenberg, R. Hidalgo-Alvarez and J. de Vicente, "Steady shear magnetorheology of inverse ferrofluids", J. Rheol. 55, 127 (2011)

Shulman Z. P., V. I. Kordonskii, E. A. Zal'tsgendler, I. V Prokhorov., B. M.Khusid and S. A. Demchuk, "Dynamic and physical properties of ferrosuspensions with a structure rearranged by an external magnetic field" Magnetohydrodynamics 20, 354-361 (1984)

Shulman, Z. P., and W. I. Kordonsky, Magnetorheological effect, Nauka i Tehnika, Minsk, 1982 (in Russian)

Stolbov O. V., Y. L. Raikher, and M. Balasoiu, « Modelling of magnetodipolar striction in soft magnetic elastomers », Soft Matter 7, 8484-8487 (2011).

Tao, R., and J. M. Sun, "Three-dimensional structure of induced electrorheological solid," Phys. Rev. Lett. 67, 398-401 (1991).

Volkova O., Cutillas S., Bossis G., "Shear banded flows and nematic-to-isotropic transition in ER and MR fluids.” Phys. Rev. Let., 82, 233-236 (1999). 
Volkova O., G.Bossis, M.Guyot, V. Bashtovoi, and A. Reks, "Magnetorheology of magnetic holes compared to magnetic particles" J. Rheol. 44, 91-104 (2000).

Volkova O, Bossis G, Lacis S., Guyot M. "Magnetorheology of a millimetric steel sphere suspension" Int.J.Mod .Physics B 16 (2002),2758-2764

Zubarev A.Yu., "On the theory of the magnetic deformation of ferrogels", Soft Matter 8, 3174-3179 (2012)

Zubarev A.Yu., and A.O. Ivanov "Nucleation stage of ferrocolloid phase separation induced by an external magnetic field”. Physica A. 251, 332-347 (1998).

Zubarev A.Yu, Iskakova L. Yu. "On the theory of rheological properties of magnetic suspensions," Physica A 382, 378-388 (2007). 


\section{Figure captions}

Fig.1. Sketch of the drop-like aggregate subjected to the shear and magnetic fields.

Fig.2. The tensile force $f$ (in arbitrary units) vs. the drop aspect ratio $r$.

Fig.3. Theoretical and experimental dependencies of the field-specific viscosity vs. the Mason number. Experimental curves represent the best fit to the power law $[\eta] \propto M a^{-v}$ of the experimental data of Halsey et al. (1992) (electrorheological fluid composed of silica particles dispersed in 4-methylcyclohexanol at $10 \% \mathrm{vol}$ and at the applied electric field of $1 \mathrm{kV} / \mathrm{m}$ ); Volkova et al. (2000) (MRS composed of carbonyl iron particles dispersed in a silicon oil at $5 \% \mathrm{vol}$ and at $7<H<20 \mathrm{kA} / \mathrm{m}$, a red circle indicates the position of a small "shoulder" of the flow curve); Klingenberg et al. (2007) (MRS composed of iron particles in hydrocarbon oil at $45 \%$ vol, we had to renormalize these data to the linear magnetization limit used in the three compared theories, such that the proportionally factor between our and Klingenberg's et al. definitions of the Mason number is $2 \chi_{s}^{2} /(9 \varphi)$, with the value $\chi_{s} \approx 4.2$ of the suspension magnetic susceptibility found form the interpolation formula proposed by the same authors for the suspension magnetization in the limit of vanishing fields)

Fig.4. Mason number dependency of the field-specific viscosity in a broad range of Mason numbers. Experimental data of Berli and de Vicente (2012) (full circles connected by a solid line) were obtained for a $10 \%$ vol suspension of carbonyl iron particles (diameter $800 \mathrm{~nm}$ ) dispersed in silicon oil without a stabilizing agent. According to Berli and de Vicente (2012), these data are perfectly fitted by Casson rheological equation written in the following form in terms of the field-specific viscosity: $[\eta]=\left(M a / M a^{*}\right)^{-1}+\left(M a / M a^{*}\right)^{-1 / 2}$, with $M a^{*}$ being an adjustable parameter. Our experimental results (symbols not connected by a line) were obtained for $5 \%$ vol. suspensions of cobalt spherical particles (diameter $1.34 \mu \mathrm{m}$ ) dispersed in silicon oil and stabilized by adsorption of aluminum stearate on particle surface. These data are regrouped onto a single master curve in the range of Mason numbers, $10^{-4}<\mathrm{Ma}<10^{-2}$; outside this range, the data deviate from the master curve. Solid lines stand for the predictions of the bulk destruction [Eq. (23a)] and erosive destruction [Eq. (23b)] models in the limit of low Mason numbers. Red dashed curve corresponds to extension of the bulk destruction model to higher Mason numbers 